\title{
Analysis on the Impact Factors for the Pulling Force of the McKibben Pneumatic Artificial Muscle by a FEM Model
}

\author{
Qin Tu $\mathbb{D}^{1},{ }^{1}$ Yanjie Wang $\mathbb{D}^{2},{ }^{2}$ Donghai Yue, ${ }^{1}$ and Frank Agyen Dwomoh $\mathbb{D}^{3}$ \\ ${ }^{1}$ School of Intelligent Equipment, Changzhou College of Information Technology, Changzhou, Jiangsu 213164, China \\ ${ }^{2}$ School of Mechanical and Electrical Engineering, Hohai University, Changzhou, Jiangsu 213022, China \\ ${ }^{3}$ School of Engineering, Koforidua Polytechnic, Koforidua, Eastern Region, Ghana \\ Correspondence should be addressed to Qin Tu; tuqin@ccit.js.cn
}

Received 23 August 2019; Revised 19 November 2019; Accepted 2 December 2019; Published 22 January 2020

Academic Editor: Yangmin Li

Copyright (c) 2020 Qin Tu et al. This is an open access article distributed under the Creative Commons Attribution License, which permits unrestricted use, distribution, and reproduction in any medium, provided the original work is properly cited.

\begin{abstract}
Modelling the behaviour of Pneumatic Artificial Muscle (PAM) has proven difficult due to its highly complicated structure, nonlinear nature of rubbery material, and air compressibility. To overcome these limitations, a FEM (Finite Element Method) model using Abaqus and CATIA is derived for the quantitative analysis on the impact of different factors on the pulling force of PAM. In the Abaqus a two parameter Mooney-Rivlin model is utilized to consider the hyper-elastic nature of flexible material. Then both Abaqus and CATIA are used in the parametric design of a 3-Dimensional model of PAM. Furthermore, the FEM model is employed to predict the static force exerted by PAM and the results show that the model is promising. The FEM model produces closer results to the test data for the typical PAM. Nonlinear behaviour of PAM is found to be obvious with an increase in both the contraction and the air pressure, different from the linear curves obtained by the fundamental geometrical model. Nonlinear changes in the PAM force are also observed in the numerical study on the effect of structural factors including initial braid angle, initial diameter, initial wall thickness, and flexible material. Besides, these phenomena can be explained by a connection between mechanical and morphological behaviour of PAMs with the FEM model. Generally, this modelling approach is more accurate compared to the fundamental theoretical model and more cost competitive compared to the empirical methods.
\end{abstract}

\section{Introduction}

The Pneumatic Artificial Muscle (PAM), sometimes termed as the McKibben Pneumatic Artificial Muscle, has been widely applied in wearable robots and rehabilitation exoskeletons for its compact structure, inherent compliance, and low price [1-3]. It consists of a cylindrical flexible tube, braided mesh shell, and two end caps $[4,5]$. While PAMs have been commercialized since 1950's, the existing types and specifications provided by different companies cannot meet the needs of different applications, not even of the joints in a single bionic robot. However, the study on the impact factors for the force exerted by PAM is rarely addressed, which encumbers the optimal design of it. Moreover, the compliance of PAM leads to the difficulty in precise control, which means there will be much work in the testing of proper air pressures of PAMs under static conditions, which hinders its further usage. Additionally, in the configuration of a rehabilitation exoskeleton, as PAM expands radially when pressurized, it is necessary to consider both how to predict the driving force of a new PAM and how much space should be left for it in installation $[1,6]$. Therefore, the modelling of mechanical behaviour of PAM is needed, as well as an analysis on the morphological behaviour. A systematic survey on the impact factors for the exerted force is also vital for the optimal design of PAMs.

In order to predict the PAM force in a static state, the modelling method evolves from the geometrical model $[4,7,8]$ and the phenomenological model $[9,10]$ to the empirical model $[11,12]$. However, the nonlinear behaviour of PAMs and the underlying mechanism are hard to describe due to the neglect in the property of rubbery materials and the accurate 3-Dimensional structure of PAMs. Moreover, the effect of air pressures and contractions rather than structural factors on the force of PAM are mostly reported, which makes it difficult to predict the mechanical and morphological performance of PAM with new structures or different materials [8]. Among different modelling techniques, the fundamental 


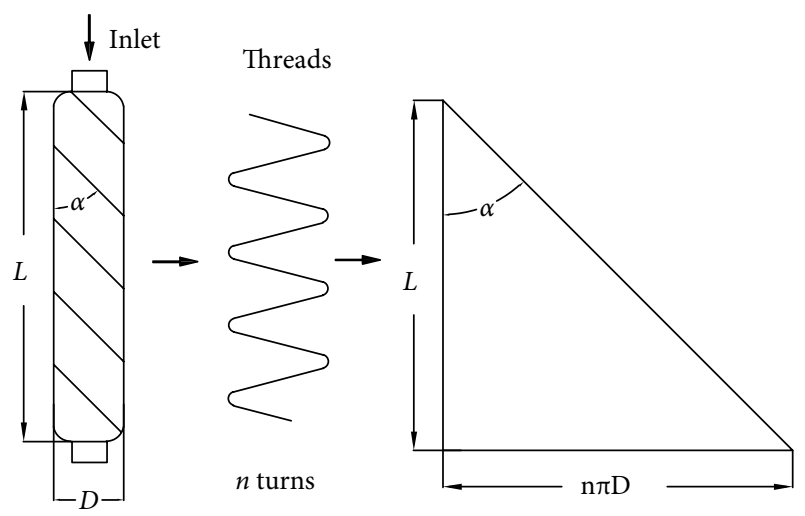

FIGURE 1: Geometry of PAM.

geometrical model, a means based on an assumption of the simplified cylindrical structure of PAM, is generally employed, and it often exhibits a relatively high deviation from the experiment $[4,7,8]$. Tsagarakis et al. [12] improved the model by considering the elasticity of rubber, and the friction effect and the distortion effect. Nevertheless, it is still hard to describe the nonlinear characteristics of PAM at lower contractions and pressures because the elastic model rather than the hyperelastic model is adopted for the rubber $[4,12,13]$. For the phenomenological model considering the behaviour of PAM as a combination of three equivalent elements including a spring, a damping, and a contraction, it fails to describe the creep phenomenon of PAM caused by the dynamic friction force $[9,14,15]$. For the empirical model, it is difficult to derive the relationship between the pressure and the displacement at no load, though in normal conditions the accuracy can be improved through the experimental identification method [13-15]. Furthermore, the empirical model requires great workload. Zang et al. [13] and Devi et al. [16] introduced the Finite Element Model (FEM) in the analysis of the characteristics of PAM considering the nonlinear material model for the rubber tube, and it generally showed better performance. However, the deviation between the simulated force and the test result is still not negligible at pressures too low or too high or at higher contractions, which may be relevant to the neglect of an accurate 3-D structure of PAM [13, 16]. Although the accuracy in the modelling of the PAM force is gradually improved by different techniques, the mechanism based on the connection between mechanical and morphological performances at varying pressures is generally ignored, which in turn hinders the improvement of these models. It also leads to the difficulty in the understanding of a higher power yielded by a pressurized PAM actuator compared to that of a traditional cylinder. Based on these, a systematic investigation on the impact factors for the actuator force of the PAM is necessary and a universal model needs to be established [17].

Considering the wide range of air pressure and size in the cases such as bionic robots or wearable robots, a simulation model of PAMs is required to set a proper pressure for a desired force and contraction for a given PAM as well as to predict the performance of PAMs with new structures. Therefore, this study focuses on the construction of a model using Abaqus and CATIA to predict the behaviour of PAMs. The effect of operating factors and structural factors on the PAM force will also be investigated in order to derive the working mechanism of PAMs for the optimal design and operation.

\section{Modelling of the Static Characteristics of PAMs}

As shown in Figure 1, the hollow tube of a PAM is usually made of rubber or silicone rubber and the threads in the braided mesh are inflexible [4]. When the air is released into one end of PAM, the tube stretches circumferentially and the other end with or without load starts to contract. The force generated depends on the air pressure, its structure, and the property of materials adopted.

2.1. Geometrical Model of PAMs. An analytic model for the mechanical behaviors of PAMs is the basis for the precise control of them in a static state. The typical modelling method is based on the geometric features of a PAM in which the effect of the braided threads is mainly taken into account. Suppose the muscle is completely cylindrical, using "virtual work" argument or energy conservation, the exerted force by a PAM actuator can be described as below $[4,18]$.

$$
F=\frac{p \pi D_{0}^{2}}{4 \sin ^{2} \alpha_{0}}\left[3 \cos ^{2} \alpha_{0}(1-\varepsilon)^{2}-1\right],
$$

where $F$ is the actuator force of PAM, $D_{0}$ is the initial muscle diameter, $\alpha_{0}$ is the initial braid angle, and $\varepsilon$ is the contraction. This fundamental equation neglects the distortion effect near the two end caps, the wall thickness, and the friction force between the flexible hose and the braided mesh. For this reason, it cannot be used in the precise prediction of PAM force for some lifting tasks $[4,18]$. Additionally, this equation does not consider the nonlinear characteristics of the rubber $[4,12]$. Nor does it include the pressure threshold to overcome the elasticity of rubber tube due to circumferential stretch and longitudinal compression. Delson et al. [19] introduced the Mooney-Rivlin equation for rubber elasticity in the derivation of a static model, and found the nonlinear material plays a more important role in the accuracy of modelling than the friction effect does. However, there is still a consistent slope error between the predicted force and experimental data at high pressures, which may attribute to the fact that the muscle diameter is not identical along the length of the tube. Through the contributions by different researchers, it is hard to incorporate all the impact factors or phenomena in an analytical model. In order to establish a general model, the Finite Element Method (FEM) which can predict the characteristics of PAM by simulating strain and stress of every element is employed. With this technique the working mechanism of PAM can be derived.

2.2. Finite Element Method. The Finite Element Method is widely used in the analysis of machining process and the development of robots $[20,21]$. Abaqus shows superiority to some other software based on FEM in dealing with the nonlinear problems, thus Abaqus combined with CATIA is 


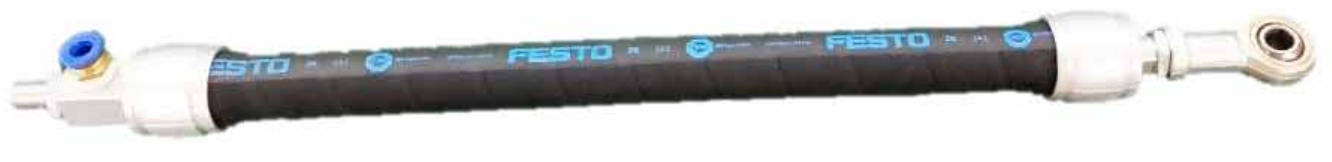

(a)

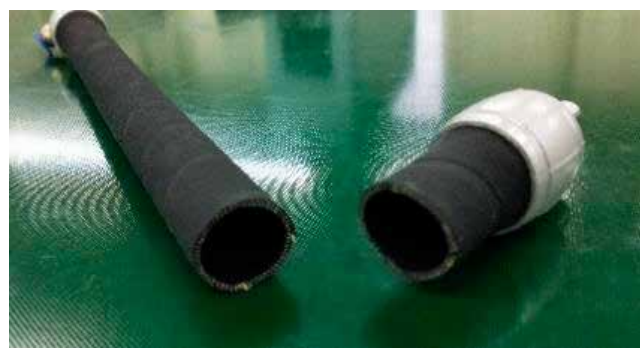

(b)

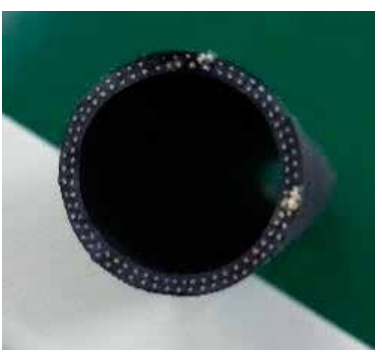

(c)

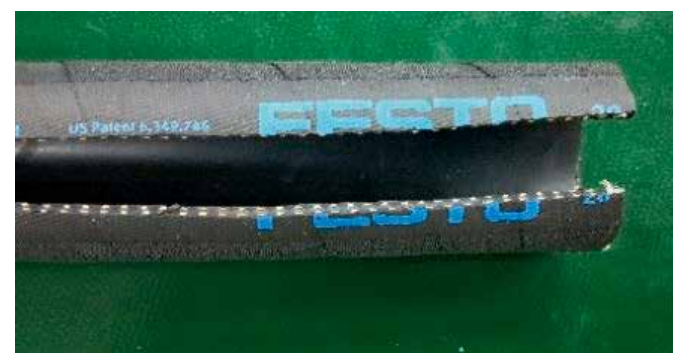

(d)

Figure 2: Structure of PAM. (a) The whole PAM. (b) Cut position. (c) Radial section. (d) Axial section.

Enlarged axial section of the tube

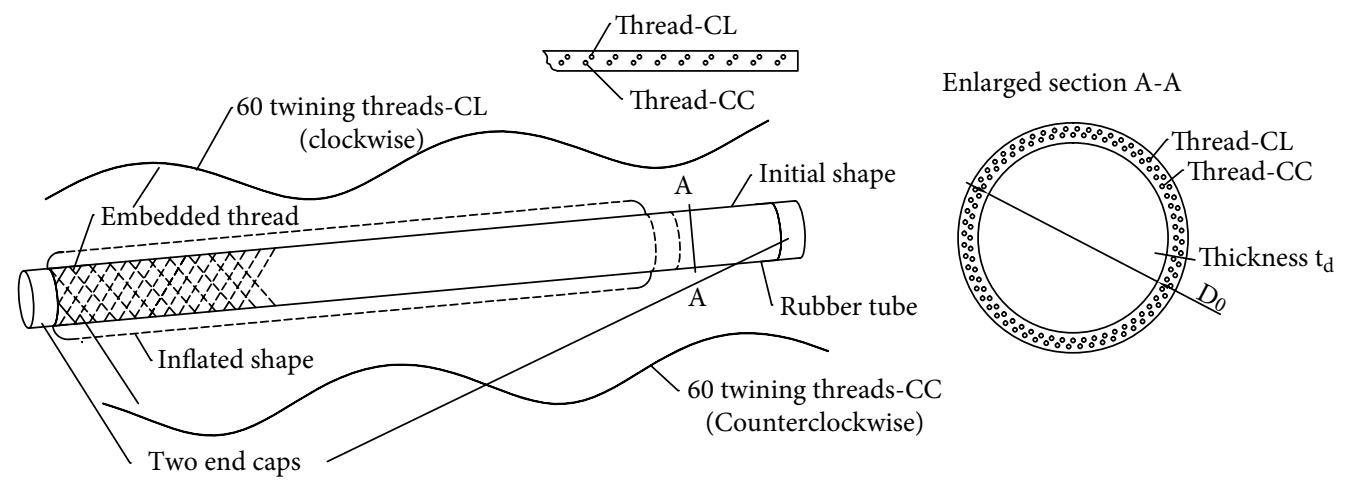

Figure 3: 3-D model of PAM.

employed in the parametric design and simulation of PAMs. Whether the accuracy of a FEM model is acceptable or not depends on three factors: a proper 3-Dimensional structure, a reasonable material model, and an effective meshing method. By doing this, all the operation factors and structural factors for the force of PAMs can be taken into account and it will establish a link between mechanical and morphological behaviour of PAM. In the previous geometrical models or FEM models, the braided threads were supposed to be coated outside the airtight tube, and the threads were thought to be contacted to each other. These assumptions also need to be verified by the experiment.

2.2.1. 3-D Structure of PAMs. In this research we use the typical McKibben muscle typed DMSP-20-300N-RM-CM with initial muscle diameter of $20 \mathrm{~mm}$, wall thickness of $2 \mathrm{~mm}$, and tube length of $300 \mathrm{~mm}$, as shown in Figure 2(a). In order to build an accurate 3-D model, a new PAM of this type is cut both axially and circularly, as shown in Figures 2(b)-2(d). In Figure 2(c) the numbers of threads on the outer layer and the inner layer are both 60. In Figure 2(d) the axial spacing of two adjacent threads for each layer is both $2.4 \mathrm{~mm}$. According to the experiment on another PAM of this type we observe that it contracts rather than twists when inflated. Then we assume that the threads are braided in two directions, clockwise (thread CL) and counterclockwise (thread CC), as shown in Figure 3. From Figure 2 we can deduce that the threads in two directions are embedded in two layers of the rubber tube. By measuring, the diameters of the two layers are $20.6 \mathrm{~mm}$ and $21.8 \mathrm{~mm}$ respectively. On the surface of the tube shown in Figure 2(a) there is a layer of twined overlapped woven cloth with an axially glued mark. In contrast, in Figure 2(c) we see no clear interface between the tube and the woven cloth. They are bonded together tightly by glue. These suggest that there is no friction between the flexible hose and the braided mesh or between the reinforcement threads $[4,22]$. As a result, the 
force balance equations in the geometrical model may need to be reconsidered $[4,12]$. In Figure 2(c), every thread in the two layers is made of several fibers. And the braid angle shown in Figure 1 can be derived with provided parameters, which is 24.8 degree.

In order to simplify the modelling of PAM behaviour with Abaqus and to reduce simulation time, two aluminium ends shown in Figure 2 are reduced to two short cylinders; the woven cloth outside is considered as part of the rubber tube, and every thread is considered as one combined wire of fiber with no thickness. The rubber tube and two end caps are constructed in Abaqus. A winding thread is built in CATIA, and then it is input, replicated and embedded radially in the rubber tube model in Abaqus. The integration of parametric design and simulation for a complicated PAM is enabled with the Combination of Abaqus and CATIA in the derivation of a 3D model enables, as shown in Figure 3.

2.2.2. Material Model of the Rubber Tube. In an improved theoretical model of PAM, a linear elastic model is usually applied for the rubber tube and the elastic modulus is set as 1.3 $\mathrm{MPa}$ [23]. However, according to Zang [23] the rubber used in the tube is Nitrile-Butadiene Rubber (NBR) chloroprene, which means a nonlinear model is required. The nonlinear models widely employed for the hyperelastic material include Mooney-Rivlin model, Yeoh model, and Ogden model $[24,25]$. Wang et al. [26] found that the Mooney-Rivlin model shows the highest agreement between the predicted performance of rubber and the test data when the tensile strain is less than $80 \%$. Since the contraction of PAM suggested by its producer Festo is $-5 \%$ to $25 \%$, the Mooney-Rivlin model is employed in the FEM model $[19,27]$. We assume that the rubber is incompressible, and then a two parameter MooneyRivlin model can be described as below [27].

$$
W=C_{10}\left(I_{1}-3\right)+C_{01}\left(I_{2}-3\right)
$$

where, $W$ is the strain energy function, $I_{1}$ and $I_{2}$ are the first and the second strain invariants of the principle stretch ratio, respectively; $C_{10}$ and $C_{01}$ are coefficients for the characteristics of rubber [23]. The parameters in Equation (2) were derived by regression according to the data in the uniaxial tensile test implemented and the results are $C_{10}=0.345083 \mathrm{MPa}, C_{01}=0.083805 \mathrm{MPa}$ for NBR chloroprene in the PAM actuator. The threads in PAM are made of polyarylamide, so we use an elastic model with the elastic modulus $2100 \mathrm{MPa}$ and the Poisson's ratio 0.3 [23]. These parameters of material, can be directly assigned to different parts of the 3D model of PAM in Abaqus. A user-defined material model based on some typical models provided in Abaqus can also be used for the original test data so that the FEM model can be improved. It is more powerful compared to an analytical model in which the values of limited variants may differ for different materials by regression.

2.2.3. Meshing Method. PAM in Figure 2 is comprised of rubber tube, two layers of threads, and two end caps. In view of the behavior of each part shown in the preliminary experiments, the two layers of threads are embedded in the rubber tube rather than considered as Rebar elements used by
Zang et al. $[22,23]$. Both the tube and the two end caps are partitioned radially and longitudinally to avoid large distortion in some elements. In the meshing process, the structured hex element and the meshing method C3D8RH are applied for the rubber tube and two end caps.

2.2.4. Boundary Conditions. In the design of a new bionic robot, the development of a proper PAM actuator for a certain joint is sometimes more urgent than the precise control of the dynamic behavior of it. In the optimal design of a new PAM the driving force and contraction are major concerns. In this work, the fixed boundary conditions are used so that it is easier to compare the static behaviour of PAM in different operation conditions and also for PAMs with different structures or different materials. Therefore, this work only discusses the static conditions including isobaric, isometric, and isotonic conditions. In these conditions, the air pressure, the load or the contraction ratio increases linearly and gradually from zero to the desired value, and the energy exchange between PAM and ambient air can be neglected, thus the accuracy in prediction may be higher. While simulating a new PAM with the FEM model, the size of winding thread can be renewed in CATIA, other structural parameters of rubber tube and end caps can be changed in Abaqus. Accordingly, the meshes in Abaqus can be regenerated. However, the loads and boundary conditions in Abaqus can maintain the same, supporting the analysis on the impact factors of PAM behaviour. For future research on the dynamic analysis, the varying pressures obtained with the experiment can be input as a user-defined load in Abaqus, and then the mechanical and morphological of PAM still can be simulated with this model. By this way, the hysteresis performance can be derived and the connection between the force output, the inflated diameter, and the stress distribution around the muscle at different time can be analyzed. In general, the FEM model can be applied to predict the behaviour of PAM in different conditions rather than used for the real-time control.

\section{Impact Factors for the Characteristics of PAM}

When a PAM actuator is used in cases such as picking small electronic products or pushing a humanoid robotic leg, the air pressure within it and the size of it may differ. In order to investigate on the characteristics of PAM in different working conditions, the force of McKibben muscle actuator at different pressures and contractions is derived with the fundamental geometrical model in Equation (1) and the FEM model. The results are compared with the experimental data provided by the producer FESTO company. From Equation (1) we can see that the impact factors for actuator force include contraction, pressure, initial braid angle, and initial diameter. From Figures 1-3 we can see that thickness and material of the tube may also need to be considered, as presented in Table 1. Two kinds of mostly applied materials used are rubber (NBR chloroprene) and silicone rubber (made with Dragon skin 30 and Ecoflex 0030 in the proportion of $1: 1$ ) [23].

Given a huge workload in the simulation of all the factors shown in Table 1 by an orthogonal test, the combination of 
TABLE 1: Impact factors for the characteristics of PAM.

\begin{tabular}{lccc}
\hline Impact factor [unit] & Value & Impact factor [unit] & Value \\
\hline Contraction $\varepsilon[\%]$ & $0,5,10,15,20$ & Initial diameter $D_{0}[\mathrm{~mm}]$ & 20,40 \\
\hline Pressure $p[\mathrm{MPa}]$ & $0.2,0.3,0.4$ & Thickness of the tube $t_{\mathrm{k}}[\mathrm{mm}]$ & $1.5,2,2.5,3$ \\
\hline & & Rubber: & $C_{10}=0.345 \mathrm{MPa}$, \\
& & $C_{01}=0.0838 \mathrm{MPa}$ & Silicone rubber: \\
Initial braid angle $\alpha_{0}$ [degree] & $17.1,24.8$ & Material & $C_{10}=0.028 \mathrm{MPa}$, \\
& & & $C_{01}=0.018 \mathrm{MPa}$ \\
\hline
\end{tabular}

these factors is considered under the normal condition. The mostly used condition (or the normal condition) for the parameter is set as: pressure of $0.3 \mathrm{MPa}$, contraction of $10 \%$, initial braid angle of 24.8 degree, initial diameter of $20 \mathrm{~mm}$, wall thickness of $2 \mathrm{~mm}$, and material of rubber. The initial length of $300 \mathrm{~mm}$ is applied for all samples. In terms of the static control, the impact of factors on the PAM behaviour is characterized by the relationship between contraction and force of PAM.

\section{Results and Discussion}

4.1. Effect of Contraction and Pressure. Figure 4 illustrates the static force-contraction relationship of a typical PAM (with initial braided angle of 24.8 degree, initial muscle diameter of $20 \mathrm{~mm}$, tube thickness of $2 \mathrm{~mm}$, and material of rubber) at varying pressures using the theoretical model in Equation (1), the established FEM model and the experiment. Stress distributions of PAM under the pressures $0.2 \mathrm{MPa}, 0.3 \mathrm{MPa}$, and $0.4 \mathrm{MPa}$ at the contraction of $10 \%$ are given in Figure 5. As shown in Figure 4, the force of PAM declines with an increase of contraction and increases with a rise in air pressure. Among three techniques, the geometrical model yields a linear relationship between force and contraction. By contrast, the simulation results show a nonlinear relationship between these two variables. Meanwhile, the simulated forces are generally lower than the theoretical results especially at a higher contraction, which is more consistent with the experimental outcome by FESTO and Zang (2012) [23]. For theoretical results, the deviation from the experimental results increases with contraction, and a higher deviation is found at higher pressures. On the contrary, the simulation results show that the deviation from the experimental results remains almost the same with an average deviation rate at $-9.9 \%$. These results indicate that the FEM model is more accurate and robust since the complicated structure and the nonlinear material of rubber are taken into account. Therefore, it can be employed to predict the effect of different factors on the behaviour of PAM. In Figure 5, the principal stress in the cylindrical area of PAM is positive under all the pressures involved, thus it behaves as expansion stress. The principal stress in the arc-shaped areas near two end caps is negative. These areas with contract stress expand gradually and it finally results in the longitudinal compression of PAM as the air pressure increases.

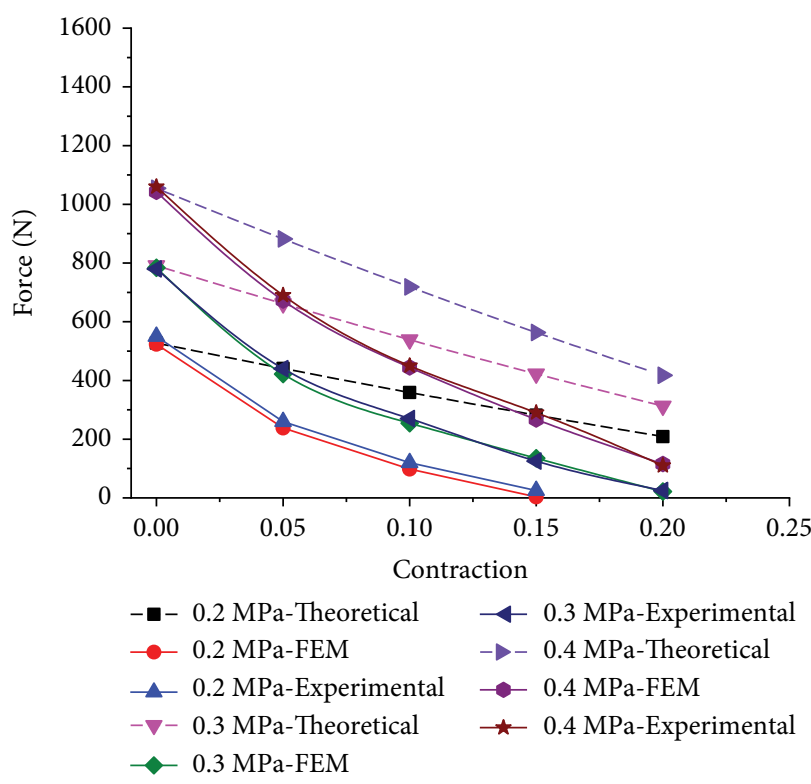

Figure 4: Static force-contraction relationship of a typical PAM at varying pressures obtained with the geometrical model, the FEM model and the experiment (isobaric condition).

The computation time is 27 minutes for the simulation of the mostly used condition on the computer with processor of Intel Core i7-8700 CPU, $3.20 \mathrm{GHz}$ and RAM of $32.0 \mathrm{~GB}$. For most cases the simulation time is within $16-40 \mathrm{~min}$, and for the following cases with silicone rubber, the simulation time is within 1.5 hour. The time cost in simulation is acceptable compared to the complicated fabrication process of different PAMs and to the conduction of an experiment.

In order to explore the impact of air pressure on the exerted force by the typical PAM, we draw the curves of force-pressure relationship at the contraction $10 \%$ obtained with three techniques in Figure 6. In addition, the comparison between initial diameter, simulated diameter, and equivalent diameter under different pressures is presented in Figure 7. The equivalent diameter here is obtained with the force divided by the corresponding pressure. For a contractor muscle, the force output is positive, behaving as a pull force when the PAM contracts. As there may be a push force at a low pressure, the equivalent diameter can be a minus. According to Figure 6, the FEM model shows an obviously closer outcome to the 


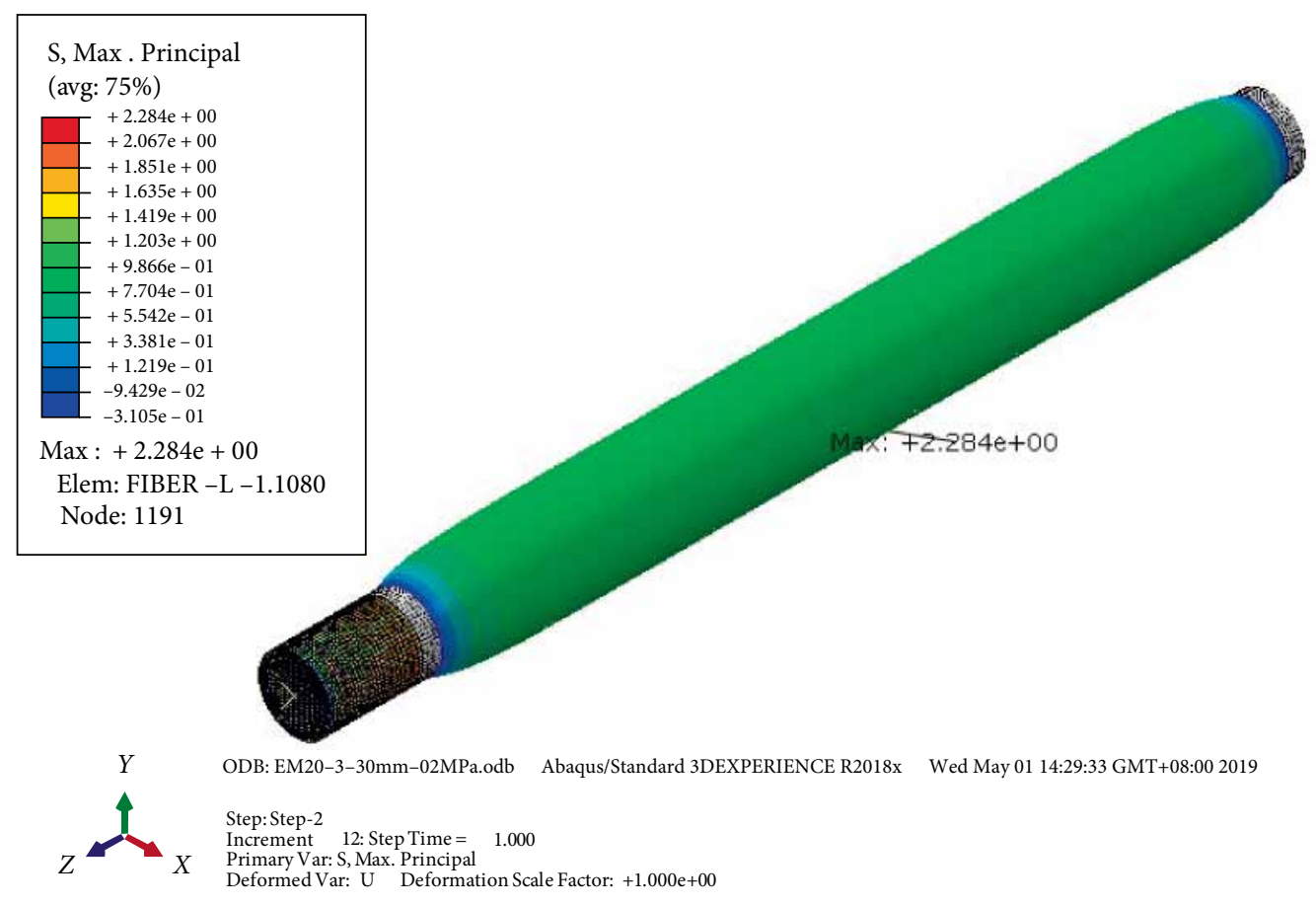

(a)

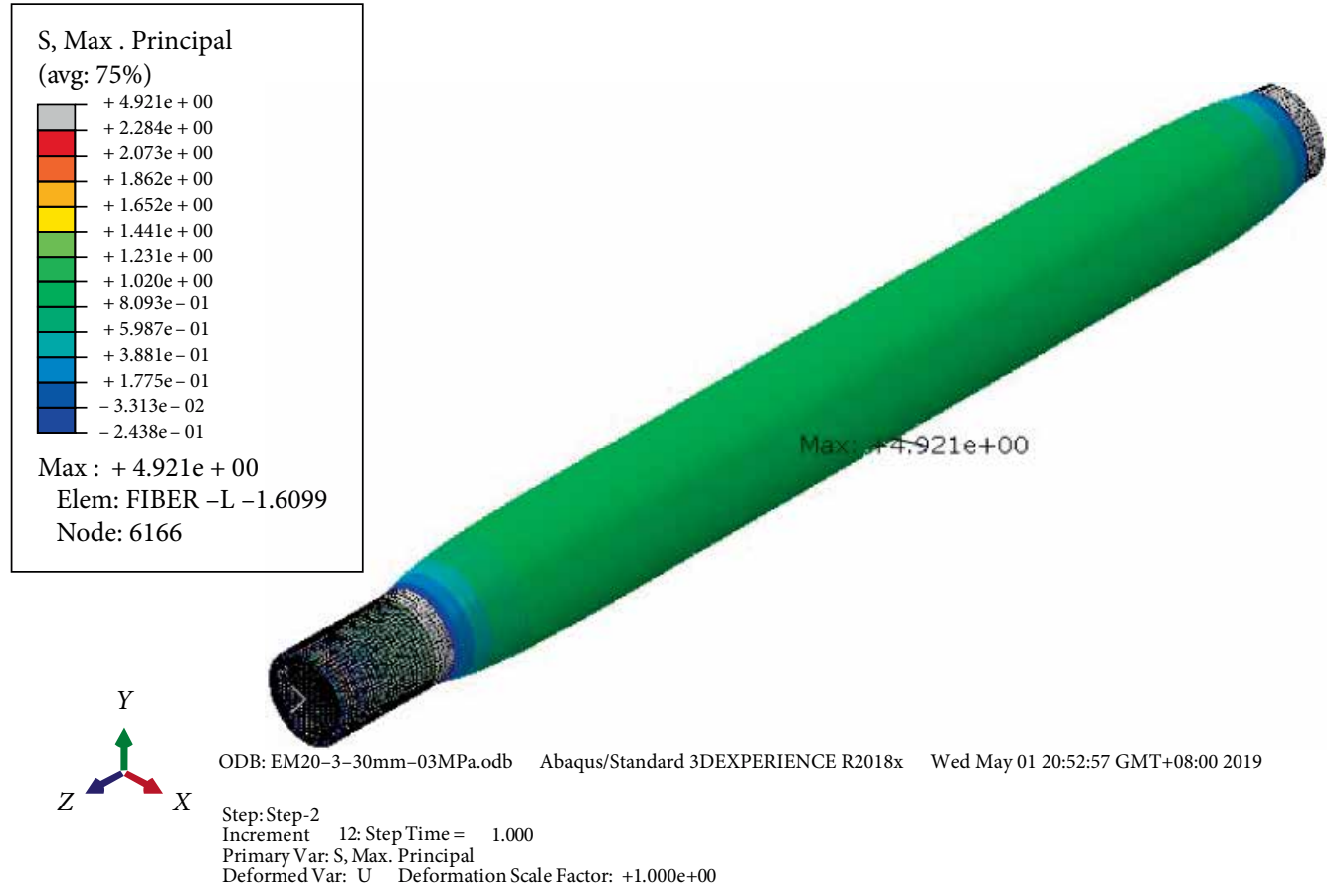

(b)

Figure 5: Continued. 


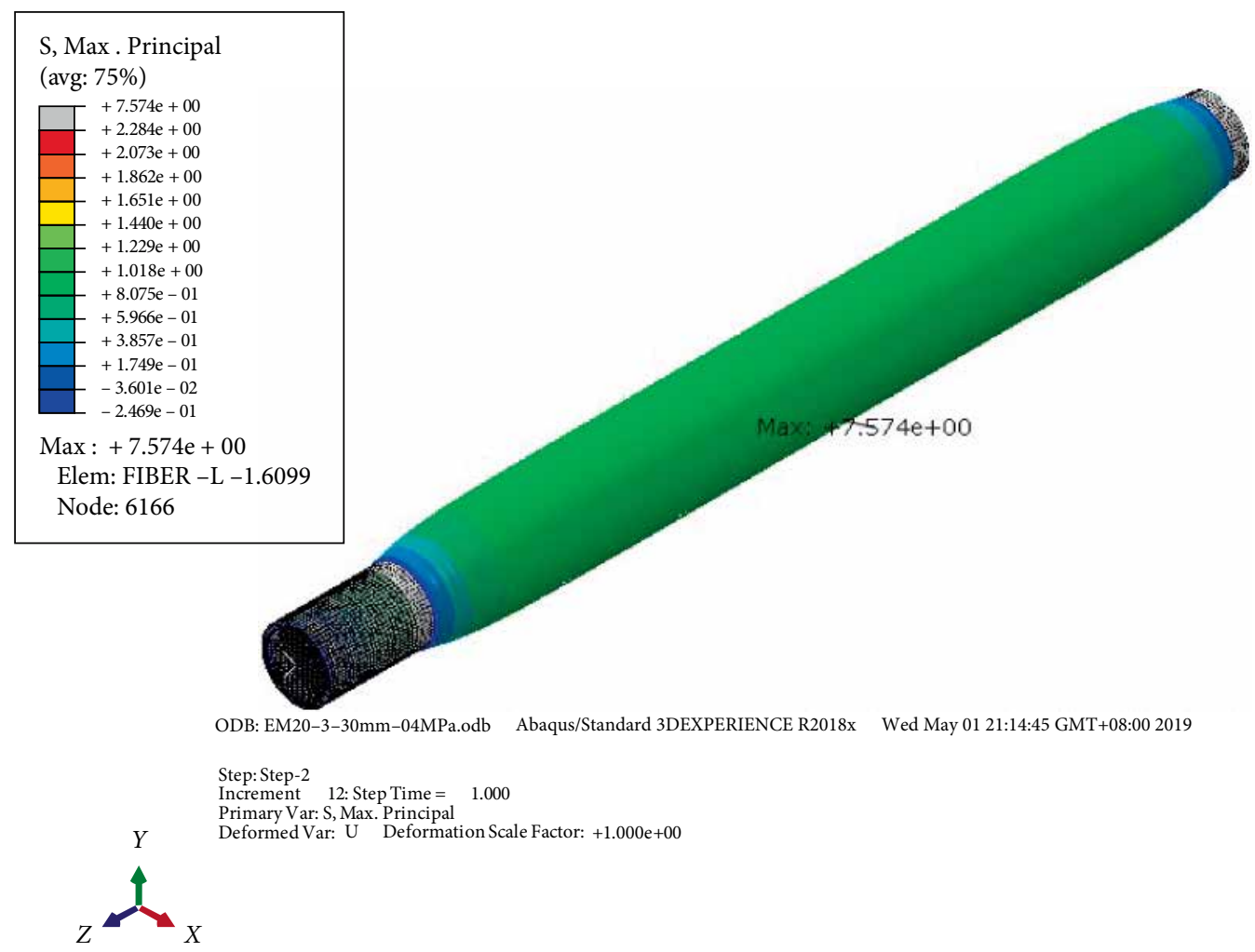

(c)

FIGURE 5: Stress distributions of PAM under the pressures of $0.2 \mathrm{MPa}, 0.3 \mathrm{MPa}$, and $0.4 \mathrm{MPa}$ at the contraction of $10 \%$. (a) $0.2 \mathrm{MPa}$, (b) $0.3 \mathrm{MPa}$, (c) $0.4 \mathrm{MPa}$.

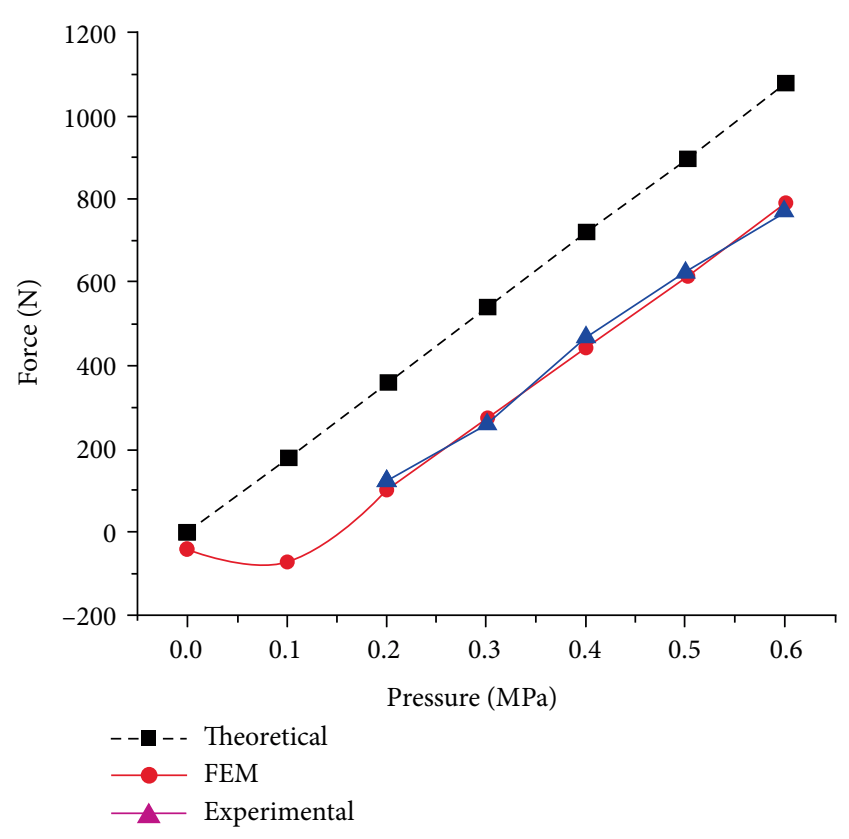

FigURE 6: Static force-pressure profile at the contraction of $10 \%$ obtained with the geometrical model, the FEM model and the experiment (isometric condition).

experimental results than the geometrical model does. When the pressure is less than $0.15 \mathrm{MPa}$, the relationship between force and air pressure obtained with the FEM model is

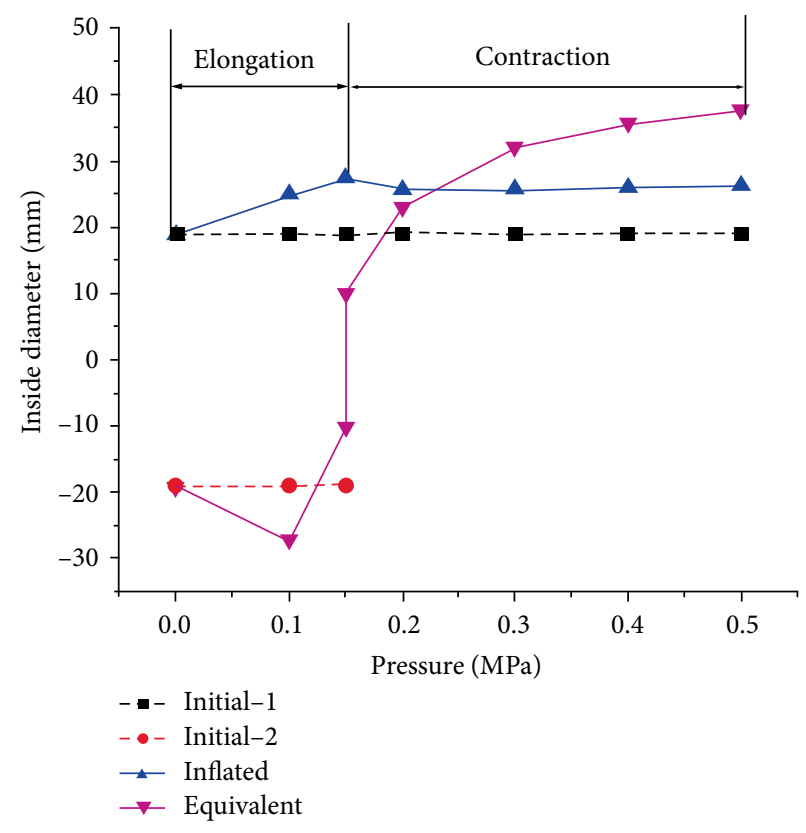

FIgURE 7: Comparison of initial diameter, inflated diameter, and equivalent diameter under different pressures at the contraction of $10 \%$.

nonlinear, the force negative, behaving as a push force. The trend is experimentally verified by Zang et al. [23]. This combined with Figures 5 and 7 can demonstrate the working 


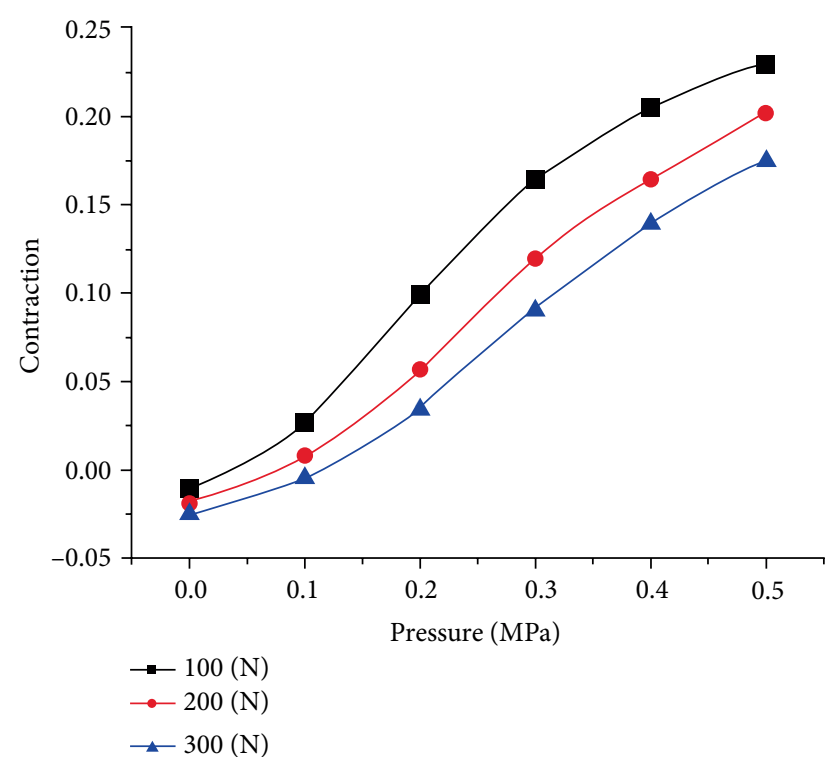

FIGURE 8: Static contraction-pressure profile under the loads of $100 \mathrm{~N}$, $200 \mathrm{~N}$, and $300 \mathrm{~N}$ (isotonic condition).

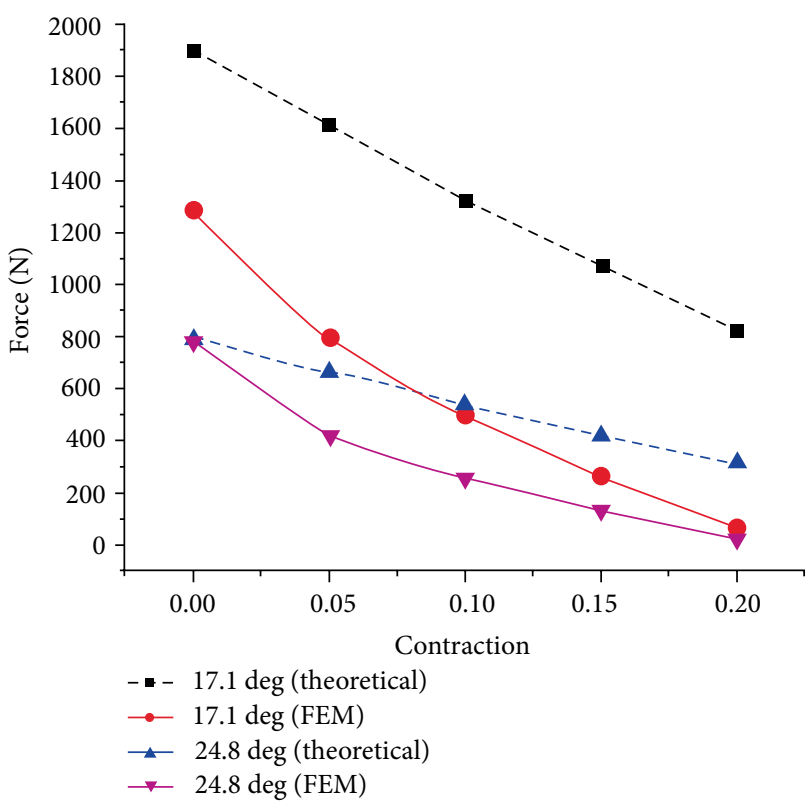

FIGURE 9: Static force-contraction relationship of PAMs with two initial braid angles of 17.1 degree and 24.8 degree under the pressure of $0.3 \mathrm{MPa}$.

mechanism of PAM. In Figure 7, when the air pressure is lower than $0.15 \mathrm{MPa}$, the simulated diameter increases sharply. The work done by pressure performs as a deformation, so the force shown in Figure 6 is low. Under this circumstance, the pressure is not high enough to overcome the radial constraint of the braided rubber tube, and the driving force behaves as a push force, as shown in Figures 5 and 6. This mechanism explains why the geometrical model is not applicable at lower pressures. When the pressure is higher than $0.15 \mathrm{MPa}$, the PAM contracts and the force performs as a pull force. The transition of muscle

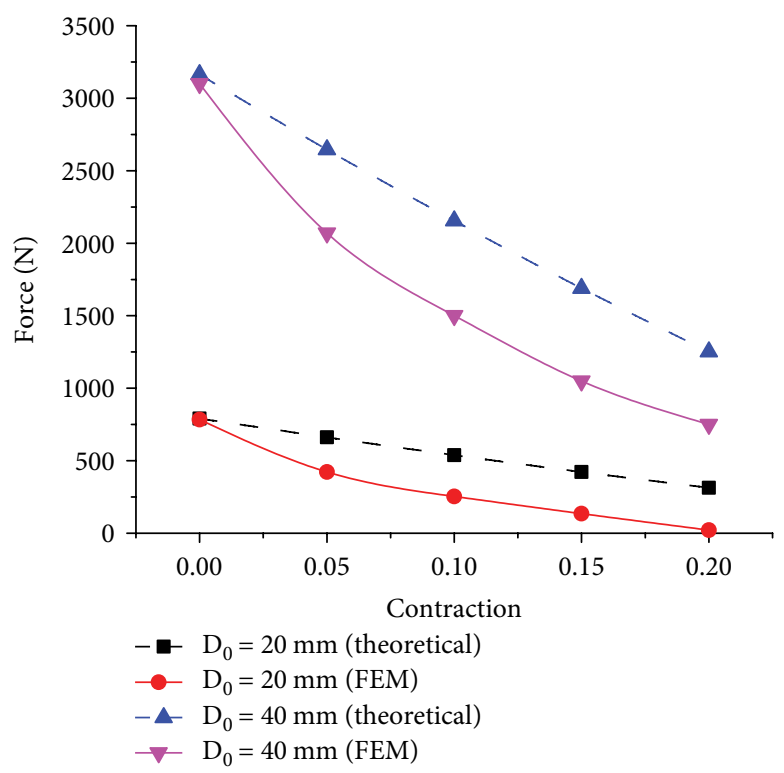

FIGURE 10: Force of PAMs with different initial muscle diameters under the pressure of $0.3 \mathrm{MPa}$.

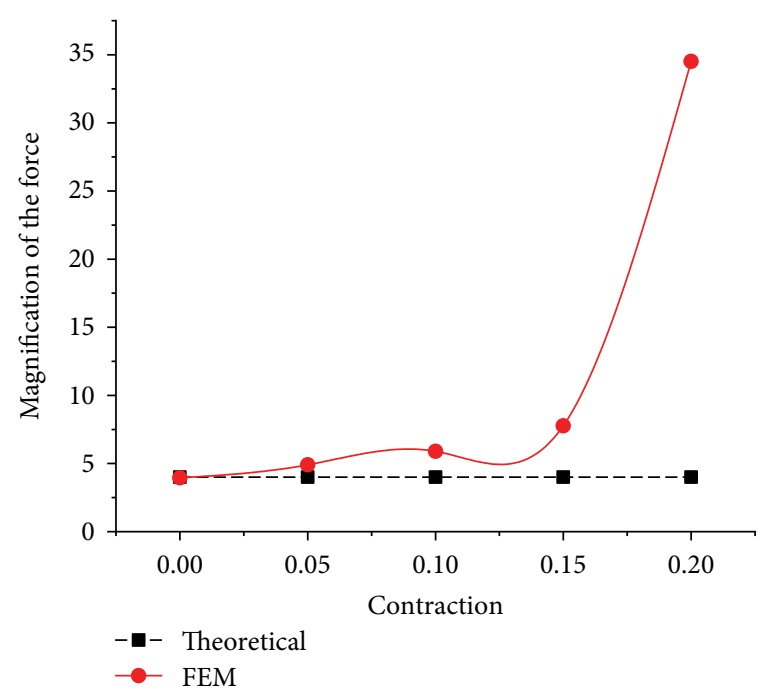

Figure 11: Magnification of force of PAM with a diameter of $40 \mathrm{~mm}$ to that of PAM with a diameter of $20 \mathrm{~mm}$.

diameter and force output around the pressure of $0.15 \mathrm{MPa}$ implies that there is a pressure threshold for the PAM force, as theoretically supposed by Chou et al. [4] and experimentally verified by Zang et al. [13] and Sangian et al. [28]. As demonstrated in Figure 7, while the inflated diameter derived by the FEM model reveals the morphological deformation of PAM, the equivalent diameter can reveal the mechanical behaviour of a PAM actuator. When the pressure is higher than $0.15 \mathrm{MPa}$, as the pressure increases, both the simulated diameter and the equivalent diameter increase, with the latter showing a larger slope. The equivalent diameter is $15.6 \%$ larger than the simulated diameter in average. The gap between these two indicators suggests the contributing effect of the braided threads since the simulated diameter is almost stable when the air 


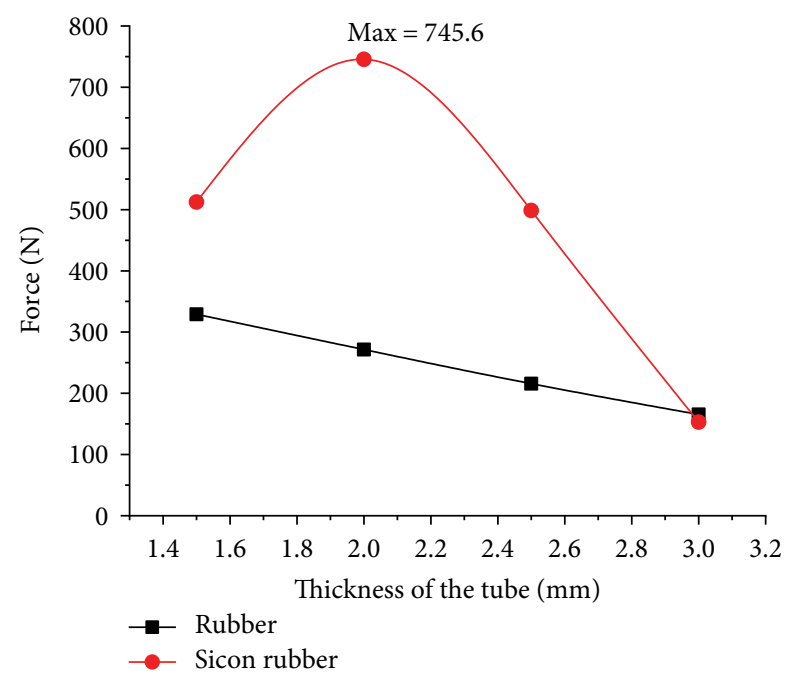

Figure 12: Force of the PAMs with different wall thicknesses and soft materials under the pressure of $0.3 \mathrm{MPa}$ at contraction of $10 \%$.

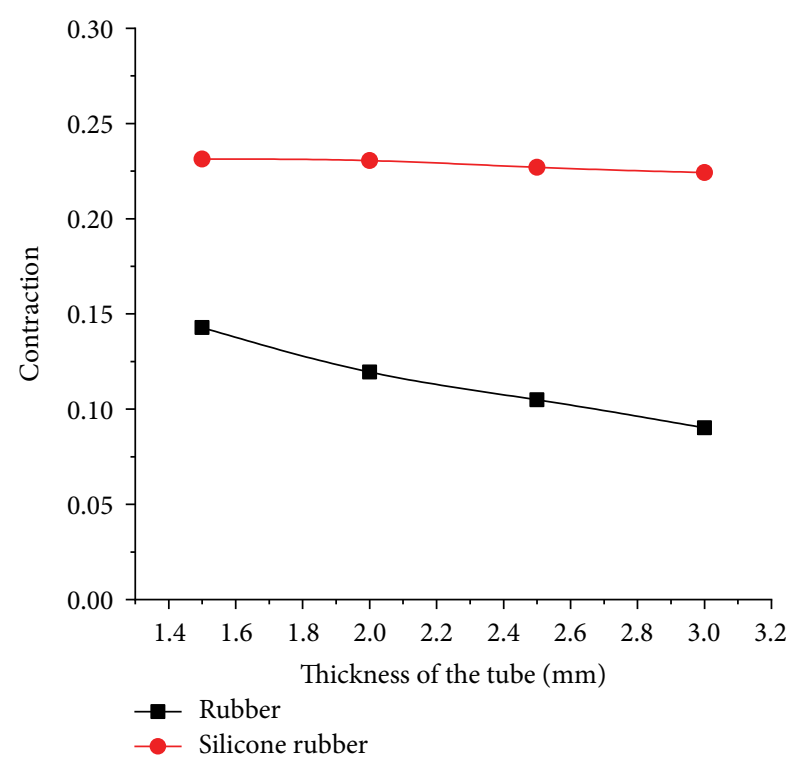

FIGURE 13: Contraction ratios of the PAMs with different wall thicknesses and materials under the pressure of $0.3 \mathrm{MPa}$ and the load of $200 \mathrm{~N}$.

pressure is higher than $0.2 \mathrm{MPa}$. This may explain why PAMs produce a higher power than traditional cylinders do.

Figure 8 illustrates the relationship between air pressure and contraction of a PAM with loads of $100 \mathrm{~N}, 200 \mathrm{~N}$, and $300 \mathrm{~N}$ using the FEM model. The results indicate that the PAM extends axially due to the load at the pressure lower than $0.1 \mathrm{MPa}$ and it starts to contract when the pressure grows higher. There is a slow increase in contraction when the pressure is lower than $0.2 \mathrm{MPa}$ and higher than $0.4 \mathrm{MPa}$, a sharp one when the pressure is between $0.2 \mathrm{MPa}$ and $0.4 \mathrm{MPa}$. The gap between contractions at different loads generally expands with the increasing pressure and narrows with the increasing load. The nonlinear trend in the contraction of PAM under different conditions is validated by the experiments conducted by different researchers $[13,18]$. This behavior in Figure 8 may be explained by an assumption that the rubber elasticity performs as a constraint to the material-induced lengthening trend at a low pressure and acts as a pressure-induced bouncing-back force at a high pressure.

4.2. Effect of the Initial Braid Angle. In the development of PAM for different applications, the braid angle is a crucial factor related to its mechanical behavior. PAMs with the initial braid angles of 17.1 degree and 24.8 degree shown in Table 1 are applied, in which the corresponding measured axial spacings of the two layers of threads shown in Figure 2 are $3.6 \mathrm{~mm}$ and $2.4 \mathrm{~mm}$, respectively. With the geometrical model in Equation (1) and the FEM model, the force-contraction relationship of PAMs with two initial braid angles at the pressure of $0.3 \mathrm{MPa}$ are demonstrated in Figure 9. From Figure 9 we can observe that a larger braid angle results in less actuator force. In the PAM with a larger braid angle shown in Figure 1, the radial inflation of rubber tube is limited, thus the equivalent diameter in Figure 7 may be reduced. Generally, the effect of initial braid angle on the force is greater at lower contractions, while the deviation between the theoretical results and the simulated results is larger at higher contractions. The force-contraction relationship of PAMs with different initial braid angles is linear obtained by the geometrical model and it is nonlinear by the FEM model yet. The force obtained with the theoretical model is obviously higher than that with the FEM model, which indicates that the geometrical model overestimates the effect of the initial braid angle. For the simulation results, when the contraction reaches $20 \%$, there seems to be an extreme value of force of PAMs with different braid angles, which may be related to the geometrical shape of PAM after inflation.

4.3. Effect of the Initial Muscle Diameter. From Equation (1) we can derive that the muscle diameter has a quadratic contribution to the pulling force of PAM. Figure 10 illustrates theoretical and simulated forces of PAMs with initial diameters of $20 \mathrm{~mm}$ and $40 \mathrm{~mm}$ under the pressure $0.3 \mathrm{MPa}$. And Figure 11 shows the magnification of the force of PAM with diameter $40 \mathrm{~mm}$ to that of PAM with diameter of $20 \mathrm{~mm}$. The results are derived in order to compare the power of a PAM with that of a traditional cylinder when the diameter increases. From Figure 10 we can see that the effect of the initial diameter on the force is greater at lower contractions. Whereas the deviation between the theoretical results and the simulated results is larger at higher contractions, which may be caused by the larger distortion in the arc-shaped areas near two end caps of the PAM at higher contractions than that at lower contractions $[8,12,16]$. In Figure 11, for the theoretical model, the force of PAM increases 4 times when the muscle diameter increases twice. For the FEM model, the magnification changes as the contraction increases. When the contraction is less than $15 \%$, the enlarging rate of the force of PAMs increases slowly around 4 , and it surges exponentially to 34.5 when the contraction increases from $15 \%$ to $20 \%$. It means that the effect of rubber elasticity and braided threads on the PAM force is greater at a higher contraction and for the PAM with a larger diameter. This may explain why the traditional modelling methods are hard to be used at higher 
contractions. Since a proper displacement is necessary for the PAM when used as an actuator, a PAM with a larger diameter is preferable in lifting tasks or jumping robots due to its higher force output magnification compared to that in a thinner one. Comparatively, a thinner PAM may be better for application in the rehabilitation robot in order to avoid muscle strain caused by too much distortion and force output of the PAM with a larger diameter.

4.4. Effect of the Thickness and the Material of the Tube. When it comes to the design of PAM, a larger thickness of braided rubber tube will either be an enhancement for the strength, or a constraint to the radial expansion of PAM. In the improved mathematical model considering the elastic force and the friction force by Tsagarakis et al. [12] the relationship between the thickness of PAM and the force was linear. Nevertheless, this relationship observed by experiment was nonlinear, therefore rubber and silicone rubber are considered, as shown in Table 1 [29]. Using the FEM model, Figure 12 illustrates the force of PAMs with different thicknesses and materials at the contraction of $10 \%$ and pressure at $0.3 \mathrm{MPa}$. Figure 13 shows the contraction of PAMs under the pressure of $0.3 \mathrm{MPa}$ and the load of 200 N. From Figure 12 we can see that the PAM made with rubber shows a linear relationship between the contraction force and the thickness of the rubber tube, whereas the PAM made with silicone rubber yields a nonlinear relationship between these two variables. This may imply that for PAM with the rubber tube, an increase in the wall thickness may serve as a constraint to the inflation of PAM. Whereas for PAM with the silicone rubber, an increase in the wall thickness shown in Figure 2 can enhance the power when the wall thickness is less than $2 \mathrm{~mm}$, and it restrains the inflation of PAM when the wall thickness is over $2 \mathrm{~mm}$. Generally, in Figures 12 and 13, the force and contraction of the PAM with silicone rubber are higher than that of the PAM with rubber, which verifies the results by Doumit et al. [8]. This may be related to the easier expansion, consequently a larger equivalent diameter for a softer material when the PAM actuator is considered as a cylinder. In Figure 13, the effects of wall thickness on the contraction of the PAMs with both materials are linear. A larger slope is found in the force of the PAM made with rubber compared to that of the PAM made with silicone rubber. In the cases concerned, when a higher force or a higher contraction is the goal in the design of PAMs, silicone rubber is suggested.

\section{Conclusions}

In order to understand the effect of different impact factors on the characteristics of PAMs required in the optimal design and usage of PAMs, we propose a FEM model with the combination of Abaqus and CATIA considering how the braided threads are embedded in the rubber tube as well as the hyper-elastic model for the rubber tube. The FEM model is then employed to analyze the effect of air pressure and contraction ratio on the actuator force of a typical PAM and the simulation results are compared with the theoretical results and experimental results. Further, the simulation model is applied to analyze the effect of structural factors including initial braid angle, initial muscle diameter, initial thickness of the tube, and flexible material on the actuator force. The analysis reveals that the FEM model is promising in the prediction of the static behaviour of PAMs with new structures, and it is more accurate and robust with the results in better agreements with the experimental results compared with the fundamental geometrical model. The nonlinear behaviour of PAM featured by nonlinear force-contraction relationship, force-pressure relationship, contraction-pressure relationship is discovered using the FEM model, though a linear behavior is obtained with the theoretical method. Generally, the geometrical model overestimates the effect of almost all the factors concerned. Based on the FEM model, the reason that why the fundamental geometrical model of PAM is not suitable for the situation of lower pressures could be derived. There is a pressure threshold for the PAM force. Under the pressures lower than $0.15 \mathrm{MPa}$, the PAM extends due to inadequate balance between low pressure and radial constraint of the rubber tube, and it contracts at pressures higher than $0.15 \mathrm{MPa}$. The effects of the braid angle and the muscle diameter on the driving force are greater at lower contractions, while the deviation between the theoretical results and the simulated results are larger at higher contractions. Meanwhile the combination of the rubber and the braided threads make the equivalent diameter much larger than the inflated diameter and contributes to a higher force of PAM compared with a traditional cylinder, especially at higher contractions. These results have built a connection between the mechanical and morphological behaviors of PAMs, which can provide a reference for the optimal design of PAMs and the potential development of new soft actuators. The established FEM model integrate the definition of material, detailed structural parameters and air pressure in the analysis of the performance of PAMs, therefore it has a high versatility. The boundary conditions can be further modified based on the experimental data to improve the accuracy of the model for different working conditions. In the further research, the effect of dynamic pressure curves and working frequency on the force of PAMs will be explored with this model and it will be compared with both a typical analytical dynamic model and experimental method so that the hysteresis behavior of PAMs can be analyzed from the perspective of mechanics and 3-D morphology.

\section{Data Availability}

The data involved in the results of this study are accessible from the corresponding author based on the readers' request.

\section{Conflicts of Interest}

The authors state that they have no conflicts of interest.

\section{Acknowledgments}

This work was supported by the General Program of Natural Science Fund for Colleges and Universities in Jiangsu Province (No. 16KJB460025), Excellent Young Faculty of Jiangsu Qinglan Project, the Six Talents Peak Project of 
Jainism Province (No. 2015-ZBZZ-027), 2018 Scientific Research Platform of Changzhou College of Information Technology (No. KYPT201801G) and the Youth Scientific Funds of Changzhou College of Information Technology (No. CXZK201706Q).

\section{References}

[1] R. A. R. C. Gopura, D. S. V. Bandara, K. Kiguchi, and G. K. I. Mann, "Developments in hardware systems of active upperlimb exoskeleton robots: a review," Robotics and Autonomous Systems, vol. 75, pp. 203-220, 2016.

[2] S. Wakimoto, J. Misumi, and K. Suzumori, "New concept and fundamental experiments of a smart pneumatic artificial muscle with a conductive fiber," Sensors and Actuators A-Physical, vol. 250, pp. 48-54, 2016.

[3] C. Yang, R. Kang, D. T. Branson, L. Chen, and J. S. Dai, "Kinematics and statics of eccentric soft bending actuators with external payloads," Mechanism and Machine Theory, vol. 139, pp. 526-541, 2019.

[4] C. P. Chou and B. Hannaford, "Measurement and modeling of Mckibben Pneumatic Artificial Muscles," IEEE Transactions on Robotics and Automation, vol. 12, no. 1, pp. 90-102, 1996.

[5] G. M. Whitesides, "Soft robotic," Angewandte Chemie (International Edition), vol. 57, pp. 2-18, 2018.

[6] H. Al-Fahaam, S. Davis, and S. Nefti-Meziani, "The design and mathematical modelling of novel extensor bending pneumatic artificial muscles (EBPAMs) for soft exoskeletons," Robotics and Autonomous Systems, vol. 99, pp. 63-74, 2018.

[7] J. Li, L. Liu, and Y. Liu, "Dielectric elastomer spring-roll bending actuators applications in soft robotics and design," Soft Robotics, vol. 6, no. 1, pp. 69-81, 2019.

[8] M. Doumit and J. Leclair, "Development and testing of stiffness model for pneumatic artificial muscle," International Journal of Mechanical Sciences, vol. 120, pp. 30-41, 2016.

[9] J. Huang, J. Qian, L. Liu, Y. Wang, C. Xiong, and S. Ri, "Echo state network based predictive control with particle swarm optimization for pneumatic muscle actuator," Journal of the Franklin Institute-Engineering and Applied Mathematics, vol. 353, pp. 2761-2782, 2016.

[10] S. Wolfen, J. Walter, D. Häufle, and S. Schmitt, "Bioinspired pneumatic muscle spring units mimicking the human motion apparatus: benefits for passive motion range and joint stiffness variation in antagonistic setups," in 25th International Conference on Mechatronics and Machine Vision in Practice (M2VIP), pp. 1-6, IEEE, US, 2018.

[11] K. C. Wickramatunge and T. Leephakpreeda, "Study on mechanical behaviour of pneumatic artificial muscle," International Journal of Engineering Science, vol. 48, no. 2, pp. 188-198, 2010.

[12] N. Tsagarakis and G. C. Darwin, "Improved modeling and assessment of pneumatic muscle actuators," in Proceedings of the IEEE International Conference on Robotics and Automation, pp. 3641-3646, IEEE, San Francisco, 2000.

[13] K. J. Zang, Y. Ma, N. Sun, Z. K. Niu, X. C. Gui, and X. P. Hu, "Study on finite element model of pneumatic artificial muscle," Advanced Materials Research, vol. 430, pp. 383-386, 2012.

[14] M. D. Doumit and S. Pardoel, "Dynamic contraction behaviour of pneumatic artificial muscle," Mechanical Systems and Signal Processing, vol. 91, pp. 93-110, 2017.
[15] C. J. Lin, C. R. Lin, S. K. Yu, and C. T. Chen, "Hysteresis modelling and tracking control for a dual pneumatic artificial muscle system using Prandtl-Ishlinskii model," Mechatronics, vol. 28, pp. 35-45, 2015.

[16] M. A. Devi, G. Udupa, and P. Sreedharan, "A novel underactuated multi-fingered soft robotic hand for prosthetic application," Robotics and Autonomous Systems, vol. 100, pp. 267-277, 2018.

[17] B. Wang, A. Mcdaid, T. Giffney, M. B. Abhari, and K. C. Aw, "Design, modelling and simulation of soft grippers using new bimorph pneumatic bending actuators," Cogent Engineering, vol. 4, no. 1, pp. 1-11, 2017.

[18] S. Ganguly, A. Garg, A. Pasricha, and S. K. Dwivedy, "Control of pneumatic artificial muscle system through experimental modelling," Mechatronics, vol. 22, pp. 1135-1147, 2012.

[19] N. Delson, T. Hanak, and K. Loewke, "Modeling and implementation of McKibben actuators for a hopping robot," in Proceedings 12th International Conference on Advanced Robotics, pp. 833-840, IEEE, US, 2005.

[20] G. Zhong, Y. Hou, and W. Dou, "A soft pneumatic dexterous gripper with convertible grasping modes," International Journal of Mechanical Sciences, vol. 153, pp. 445-456, 2019.

[21] Z. S. Sun, Z. H. Guo, and W. Tang, "Design of wearable hand rehabilitation glove with soft hoop-reinforced pneumatic actuator," Journal of Central South University, vol. 26, no. 1, pp. 106-119, 2019.

[22] D. H. Chen and K. Ushijima, "Prediction of the mechanical performance of McKibben artificial muscle actuator," International Journal of Mechanical Sciences, vol. 78, pp. 183-192, 2014.

[23] K. Zang, Mechanism and design theory research of braided type Pneumatic Artificial Muscle, Northeast Forestry University, Harbin, P.R. China, 2012.

[24] P. Martins, R. Jorge, and A. Ferreira, "A comparative study of several material models for prediction of hyperelastic properties: application to silicone-rubber and soft tissues," Strain, vol. 42, no. 3, pp. 135-147, 2006.

[25] R. W. Ogden, "Large deformation isotropic elasticity-on the correlation of theory and experiment for incompressible rubberlike solids, Proceedings of the Royal Society of London. Series A," Mathematical and Physical Sciences, vol. 326, no. 1567, pp. 565-584, 1972.

[26] G. Wang, M. Liu, Y. Yao, L. Jiang, and W. Duan, "Application of different constitutive models in the nonlinear finite element method for rubber parts," Mechanics in Engineering, vol. 8, pp. 40-47, 2013.

[27] E. Ball, Y. Lin, and E. Garcia, "Characterization and modeling of geometric variations in McKibben pneumatic artificial muscles," Proceedings of SPIE-The International Society for Optical Engineering, vol. 8686, p. 868605, 2013.

[28] D. Sangian, S. Naficy, G. M. Spinks, and B. Tondu, "The effect of geometry and material properties on the performance of a small hydraulic McKibben muscle system," Sensors and Actuators A-Physical, vol. 234, pp. 150-157, 2015.

[29] L. Chen, C. Yang, H. Wang, D. T. Branson, J. S. Dai, and R. Kang, "Design and modelling of a soft robotic surface with hyperelastic material," Mechanism and Machine Theory, vol. 130, pp. 109-122, 2018. 


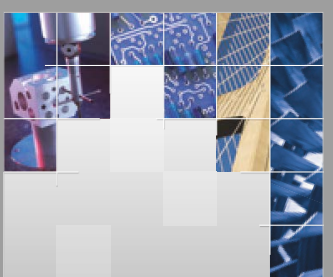

\section{Enfincering}
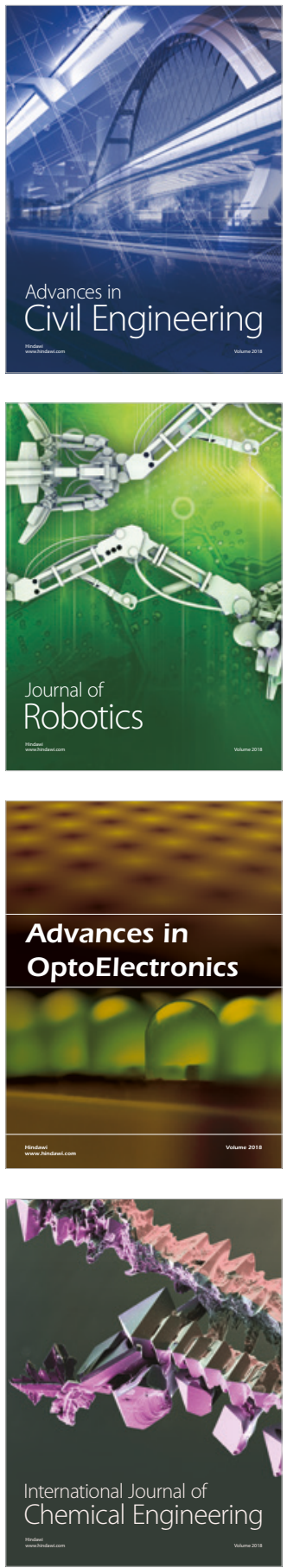

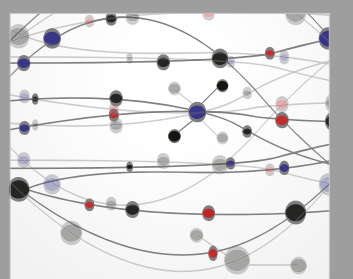

\section{Rotating \\ Machinery}

The Scientific World Journal

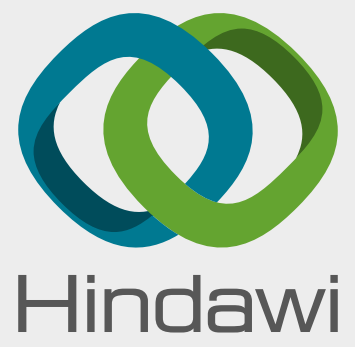

Submit your manuscripts at

www.hindawi.com
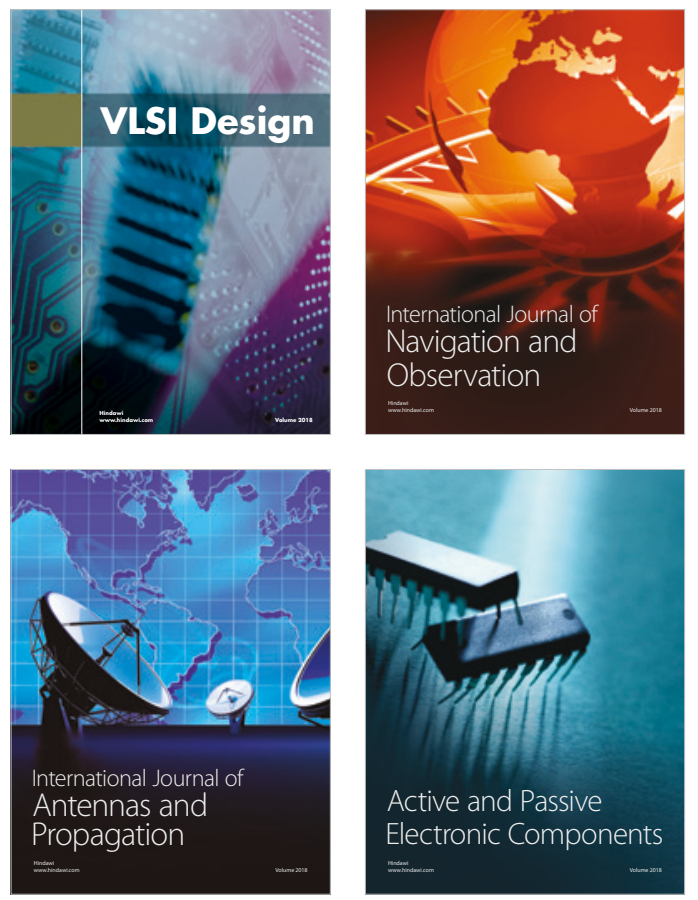
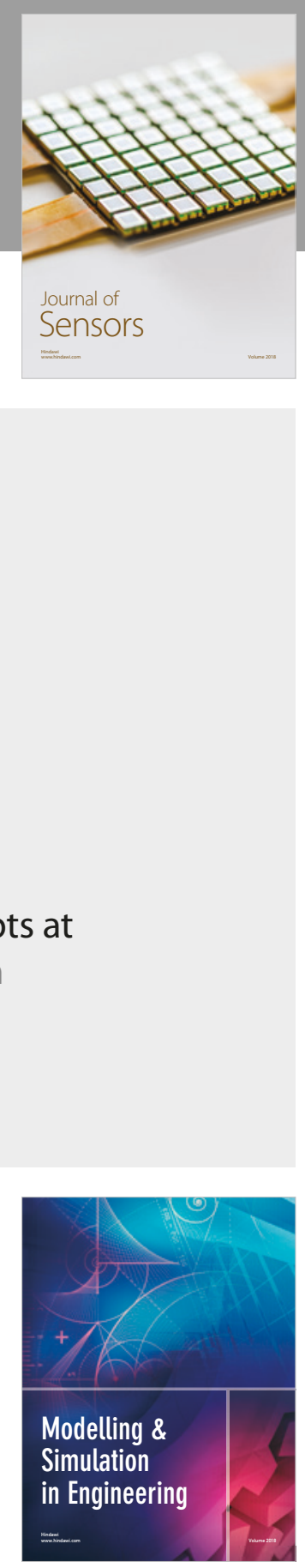

\section{Advances \\ Multimedia}
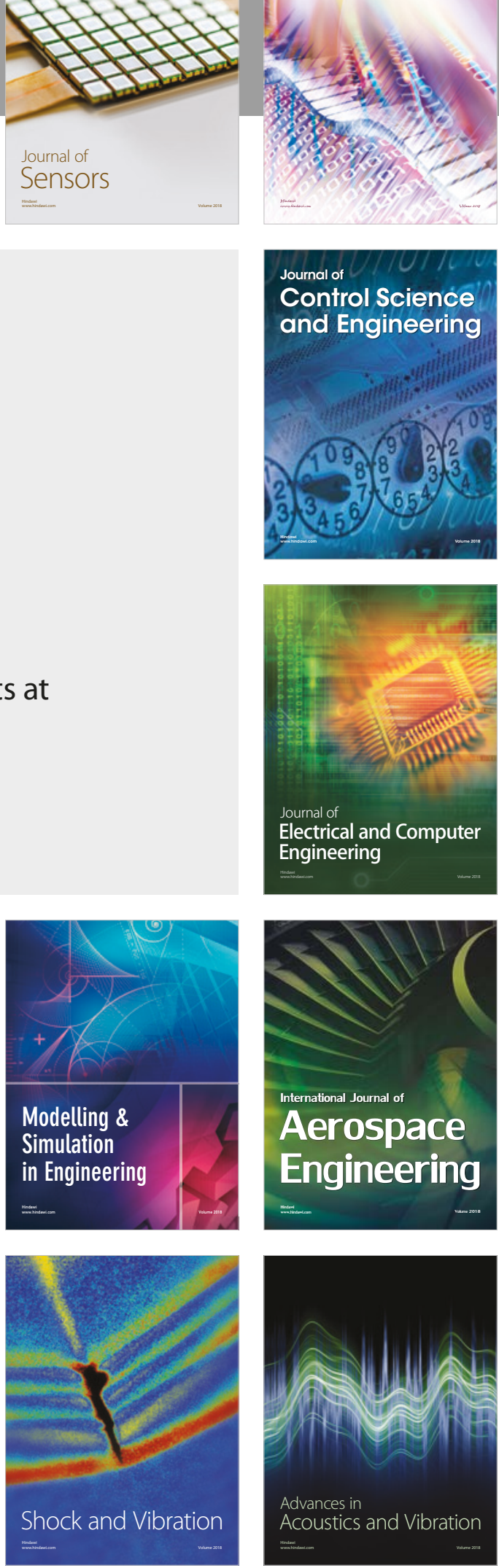